S09.02 figure 1 suggests local elimination is likely to occur earlier in Bellary (median: 2023) than in Mysore (2028) and Belgaum (2030), with the required intervention duration being $11-35$ years. The discounted cost of achieving local elimination in each of the settings is estimated to be $\$ 8-11000000$ with 5000-11000 HIV infections averted up to 2050 .

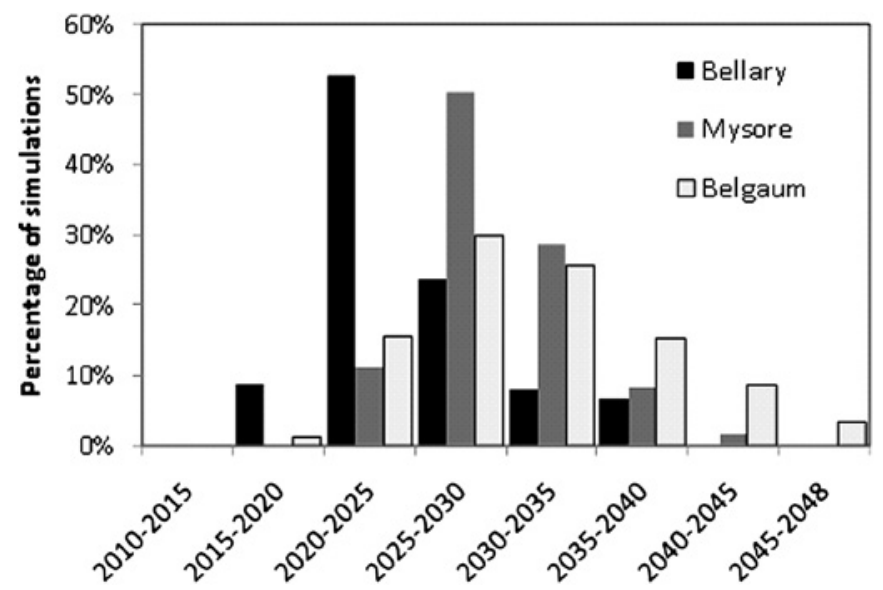

Abstract 01-S09.02 Figure 1 Posterior distribution of the year when HIV indicates in FSWs and clients goes below 1 infection per 1000 FSWs and clients.

Conclusion Our results suggest Avahan could result in local elimination of HIV among FSWs and clients in these districts without ART. Current discussions around the use of ART for HIV elimination should also consider other prevention strategies, especially in concentrated epidemic settings where eliminating HIV from FSWs and clients is likely to eliminate HIV in the general population. Our modest estimated costs for local elimination could be completely offset against averted ART costs.

\section{1-S09.03 MAIN RESULTS AND IMPACT ANALYSIS OF ANNUAL CHLAMYDIA SCREENING IN A LARGE REGISTER-BASED PROGRAMME IN THE NETHERLANDS}

\section{doi:10.1136/sextrans-2011-050109.51}

${ }^{1}$ I V F van den Broek, ${ }^{2} \mathrm{~J}$ A N E A M van Bergen, ${ }^{3} \mathrm{H}$ S A Fennema, ${ }^{4} \mathrm{H}$ M Götz, ${ }^{5} \mathrm{C}$ J P A Hoebe, ${ }^{6} \mathrm{E}$ Over, ${ }^{7} \mathrm{M}$ A B van der Sande, ${ }^{8} \mathrm{~B}$ V Schmid, ${ }^{8} \mathrm{E}$ L M Op de Coul, ${ }^{9}$ on behalf of the CSI-Group. ${ }^{1}$ Centre for Infectious Diseases Control, National Institute of Public Health and the Environment, Bilthoven, Netherlands; ${ }^{2}$ STI AIDS Netherlands, Amsterdam, Netherlands; ${ }^{3}$ Cluster of Infectious Diseases, Department of Research, Online Research and Prevention Unit, Amsterdam Health Service, Amsterdam, Netherlands; ${ }^{4}$ Division of Infectious Disease Control, Rotterdam Rijnmond Public Health Service, Rotterdam, Netherlands; ${ }^{5}$ Department of Infectious Diseases, South Limburg Public Health Servic, Geleen, Netherlands; ${ }^{6}$ Centre for Prevention and Health Services Research, National Institute of Public Health and the Environment, Bilthoven, Netherlands; ${ }^{7}$ Centre for Infectious Disease Control, National Institute of Public Health and the Environment and Julius Center for Health Sciences and Primary Health Care, University Medical Centre Utrecht, Bilthoven, Netherlands; ${ }^{8}$ Epidemiology and Surveillance Unit, Centre for Infectious Disease Control, National Institute of Public Health and the Environment, Bilthoven, Netherlands; ${ }^{9}$ Netherlands

Background Chlamydia screening programmes can only work when they motivate sufficient persons at risk to get tested regularly. The outcomes of the Chlamydia Screening Implementation (CSI) in the Netherlands are novel and valuable because of the large scale of the programme (315000 young persons targeted), its systematic nature (invitations based on municipal registers) and its multiple screening rounds (annual invitations in three consecutive years).
Methods From April 2008 to March 2010, Chlamydia Screening was offered annually to municipal-registered $16-29$ year olds in three regions of the Netherlands. A phased implementation was applied by grouping clusters in three random, risk-stratified blocks. Participation and positivity rates were compared between blocks submitted to 1, 2 or 3 screening rounds (3rd round only partially completed). The effect of the repeated screening rounds on the prevalence of Chlamydia in the whole target group was estimated by weighting procedures comparing demographic characteristics of participants and target group.

Results The participation rate in the first round was 16\% among all invitees and $20 \%$ among the sexually active target population (M13\%, F 25\%). The participation fell down to $11 \%$ in blocks invited two times and 9\% in the block invited three times, whereas it was $13 \%$ in the control block invited in round two only. The positivity rate in round one was $4.2 \%$ among all participants. Positivity rates decreased significantly to $4.0 \%$ in blocks screened twice and to $3.5 \%$ in the block screened thrice $(p=0.04)$; in the control block $4.3 \%$ tested positive. The population prevalence was estimated at 2.6\% in Amsterdam, 3.8\% in Rotterdam and 2.4\% in South Limburg. The prevalence declined over the three screening rounds, but declines were not significant (see Abstract O1-S09.03 figure 1).

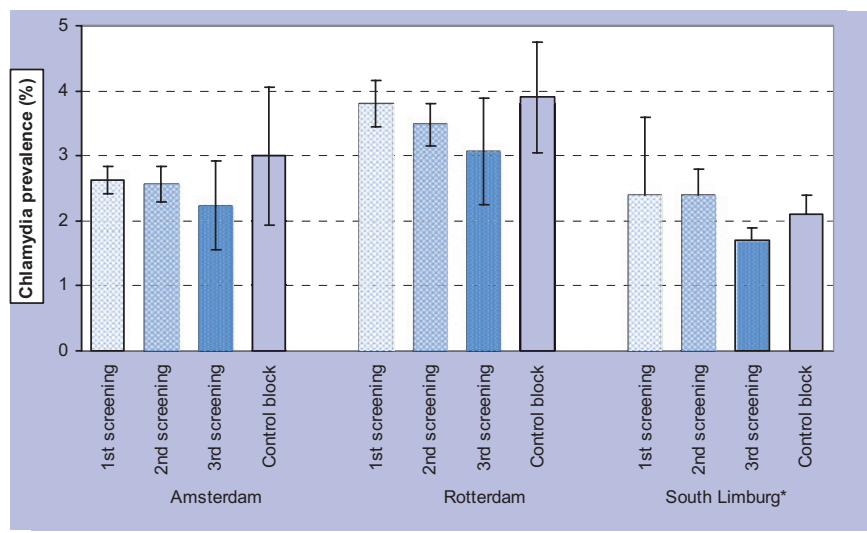

Abstract 01-S09.03 Figure 1 Estimated Chlamydia prevalence among young people invited for Chlamydia screening 1, 2 or 3 times at annual intervals, as compared to a control group invited only once. [ ${ }^{*}$ bars indicate high-low estimates for South Limburg, an adaptation to correct for participant selection.

Conclusions The participation in the CSI project was lower than expected and declined with repeated invitation. Chlamydia positivity rates were reduced by $17 \%$ in clusters screened three times, whereas these stayed high in control groups. Only a small and nonsignificant impact on population prevalence was measured. Further extrapolation of the findings in a simulation model suggest a limited impact on Chlamydia prevalence after 10 years of screening and estimates of cost-effectiveness do not support a nationwide roll-out of this programme in the Netherlands.

\section{1-S09.04 MODELLED IMPACT OF CHANGING PARTICIPATION RATES ON EFFECTIVENESS OF POPULATION BASED CHLAMYDIA SCREENING}

doi:10.1136/sextrans-2011-050109.52

${ }^{1}$ B V Schmid, ${ }^{2}$ V F van den Broek, ${ }^{2} E$ L M Op de Coul, ${ }^{3}$ J E A M van Bergen, ${ }^{4} \mathrm{~J} S \mathrm{~S}$ Fennema, ${ }^{5} \mathrm{H} \mathrm{M}$ Götz, ${ }^{6} \mathrm{C}$ J P A Hoebe, ${ }^{7} \mathrm{M}$ Kretzschmar. ${ }^{1}$ National Institute for Public Health and the Environment RIVM, Bilthoven, Netherlands; ${ }^{2}$ National Institute for Public Health and the Environment, Bilthoven, Netherlands; ${ }^{3}$ STI AIDS Netherlands, Amsterdam, Netherlands; ${ }^{4}$ Amsterdam Public Health Service, Amsterdam, Netherlands; ${ }^{5}$ Rotterdam Rijnmond Public Health Service, Rotterdam, Netherlands; ${ }^{6}$ South Limburg 